\title{
Cotyledonoid dissecting leiomyoma with intravascular growth pattern and intra-tumoural endometrial glands and stroma: A case report
}

\author{
Khurshid Merchant ${ }^{* 1}$, Bernard Chern ${ }^{2}$, Sung Hock Chew ${ }^{1}$ \\ ${ }^{1}$ Department of Pathology and Laboratory Medicine, KK Women's and Children's Hospital, Singapore \\ ${ }^{2}$ Department of Obstetrics and Gynaecology, KK Women's and Children's Hospital, Singapore
}

Received: August 23, 2017

Accepted: October 26, 2017

Online Published: November 9, 2017

DOI: $10.5430 /$ crcp.v4n4p1

URL: https://doi.org/10.5430/crcp.v4n4p1

\begin{abstract}
Cotyledonoid dissecting leiomyoma (CDL) is a rare variant of leiomyoma which shows a dissecting growth pattern of smooth muscle cells within the myometrium, with or without extra-uterine extension, and a macroscopic appearance resembling placenta. We present a unique case of CDL with both intravascular growth and presence of endometrial glands and stroma within the tumour. A 35-year-old female patient presented with menorrhagia. Ultrasound examination revealed a bulky anteverted uterus with a prolapsed submucosal leiomyoma and an intramural leiomyoma. In the interest of preserving fertility, she underwent myomectomy together with laparoscopic resections of the uterine wall deposit and myometrial tissue. The tumour was soft and pink, with a resemblance to placental tissue, and histology demonstrated nodules of neoplastic smooth muscle cells with areas of oedema and myxoid change dissecting into the surrounding myometrial tissue, which is consistent with the features of CDL. Also seen were non-neoplastic endometrial glands cuffed by stroma located within the smooth muscle tumour as well as in the non-neoplastic myometrium. There were foci of intravascular luminal growth confirmed by positive CD31 immunohistochemical staining of the endothelial cells lining the vascular lumina. The present case demonstrates that CDLs may have a unique histological appearance resembling adenomyoma and an intravascular growth pattern. It is important for pathologists, gynaecologists and radiologists to recognize such smooth muscle tumour variants and hence prevent misdiagnosis of malignancy and overtreatment.
\end{abstract}

Key Words: Cotyledonoid, Dissecting leiomyoma, Adenomyosis, Intravascular growth

\section{INTRODUCTION}

Uterine benign smooth muscle tumours can have unusual and uncommon growth patterns which macroscopically or microscopically mimic invasive tumours. Familiarity with these patterns and their recognition can be helpful in avoiding misdiagnosis and overtreatment. In addition, these tumours may feature degenerative changes like hydropic and myxoid change which can impose further difficulties in diagnosis. Cotyledonoid dissecting leiomyomas (CDLs) of the uterus are rare smooth muscle neoplasms characterized by dissecting growth patterns of smooth muscle cells with or without extrauterine extension, macroscopically resembling the maternal face of the placenta. These neoplasms present clinically and radiologically as apparently malignant growth ${ }^{[1]}$ with an age group distribution ranging from 23 to 73 years. ${ }^{[2]}$ The most common clinical presentation is abnormal uterine bleeding. ${ }^{[2]}$

*Correspondence: Khurshid Merchant; Email: khurshid.merchant@singhealth.com.sg; Address: Department of Pathology and Laboratory Medicine, KK Women's and Children's Hospital, 100 Bukit Timah Road, Singapore 229899, Singapore. 


\section{CASE PRESentation}

\subsection{Clinical presentation}

A 35-year-old female patient presented with history of prolonged and painful menses since 2 months. The patient was admitted to the gynaecology department at our hospital for further assessment and treatment. An ultrasound of the pelvis showed an anteverted bulky uterus with thickened endometrium measuring $30 \mathrm{~mm}$ in thickness and a heterogenous vascular structure measuring $10.4 \mathrm{~cm} \times 5.1 \mathrm{~cm} \times$ $4.2 \mathrm{~cm}$ in dimension within the endocervical canal, likely representing a prolapsed submucosal leiomyoma. A $1.3 \mathrm{~cm}$ $\times 1.1 \mathrm{~cm} \times 0.6 \mathrm{~cm}$ intramural fibroid was noted in the right wall of the uterus. As she desired to preserve fertility, a laparoscopic myomectomy was planned. However, at surgery a $10 \mathrm{~cm}$ mass arising from the right ovarian ligament was seen. It had an exophytic component of bulbous (cotyledonoid) smooth muscle, grossly resembling placental tissue protruding from the uterine surface (see Figures 1-2). In view of the possibility of malignancy, a mini-laparotomy was performed and the patient underwent resection of the right adnexal mass, dilatation and curettage with hysteroscopy and hydrotubation. The excised tissue was sent for frozen section evaluation.

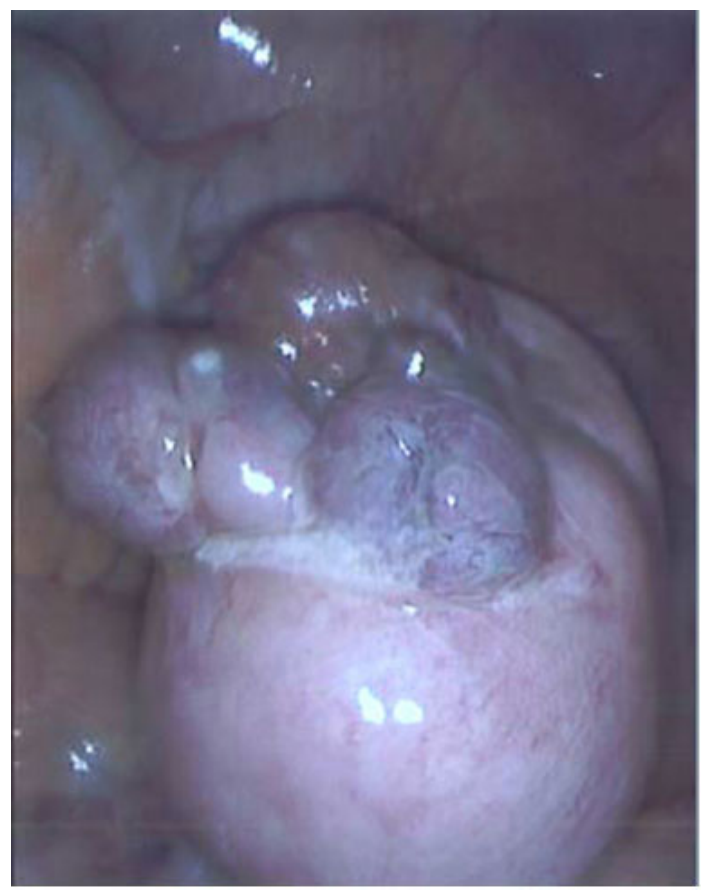

Figure 1. Intraoperative view showing placenta-like appearance of the tumour which has infiltrated through the uterine wall onto the serosal surface

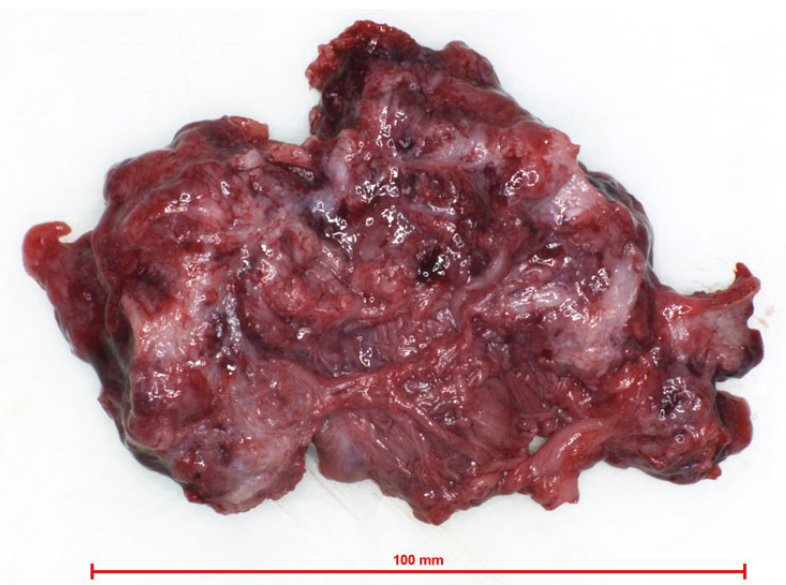

Figure 2. Macroscopic appearance of the tumour, showing a placenta-like appearance

\subsection{Pathological findings}

Altogether, four specimens were examined at the histopathology laboratory. Specimen A, labelled "fibroid", comprised several irregular fragments of pink soft tissue measuring $60 \mathrm{~mm} \times 50 \mathrm{~mm} \times 22 \mathrm{~mm}$, weighing 32 grams and showed areas of whorling and haemorrhage on the cut surface. Specimen B, labelled "uterine wall deposit", consisted of pieces of soft tissue with a pink cut surface altogether weighing 10 grams and measuring $40 \mathrm{~mm} \times 30 \mathrm{~mm} \times 5 \mathrm{~mm}$. Specimens $\mathrm{C}$ and $\mathrm{D}$ were labelled myometrial tissue. They consisted of several pieces of pinkish soft tissue with areas of congestion and measured $30 \mathrm{~mm} \times 20 \mathrm{~mm} \times 5 \mathrm{~mm}$ and $100 \mathrm{~mm} \times 60 \mathrm{~mm} \times 35 \mathrm{~mm}$ in maximum dimensions, respectively. These specimens weighed 5 and 89 grams respectively. The larger of these showed irregular dissecting margins of the tumour into surrounding myometrium (see Figure 3). These four specimens were subjected for frozen section and were reported to be spindle cell lesions with smooth muscle differentiation. The specimen labelled fibroid (A) showed occasional endometrial type glands in addition, while a possibility of dissecting leiomyoma was raised for the specimen labelled myometrial tissue (D), as islands of smooth muscle tumour were seen in between bundles of normal myometrial smooth muscle.

Histologically, Specimens A, B and C were composed of interlacing fascicles of smooth muscle bundles. Areas of haemorrhage and infarction were present. Histology also showed areas of oedema, hyaline degeneration and myxoid change. A few endometrial glands were noted on the surface in keeping with the submucosal location of the fibroid. Specimen D, labelled myometrial tissue, showed smooth muscle tumour with surrounding myometrial tissue. The tumour was histologically similar to that described for specimens A to $\mathrm{C}$, showing areas of oedema and myxoid change. Nodules 
that contained swirled neoplastic smooth muscle cells were seen dissecting into the myometrial tissue, forming broad bands and trabeculae surrounded by myometrial smooth muscle bundles. The myocytes appeared uniform with bland spindle-shaped nuclei and eosinophilic cytoplasm. Focally, intravascular luminal growth was seen within myometrium beyond the edge of the tumour, consistent with intravascular leiomyomatosis (see Figure 4). An immunohistochemical stain for CD31 highlighted the endothelial cells of the blood vessels which contained the foci of intravascular growth of the leiomyoma (see Figure 5). Occasional foci showed endometrial glands with surrounding stroma within the tumour (see Figure 6) and in the surrounding myometrial smooth muscle bundles. Immunohistochemical studies showed that the spindle cells were positive for desmin and caldesmon and negative for CD 10, supporting the smooth muscle nature of the tumour against an endometrial stromal tumour. In the islands of endometrial glands and stroma, the stromal cells were positive for CD 10, and negative for desmin and caldesmon, supporting their endometrial stromal nature. These findings support the predominant smooth muscle nature of the tumour, in keeping with dissecting leiomyoma, and exclude a low-grade endometrial stromal sarcoma (see Figures 7-8). Mitotic count was up to 1 mitosis per 10 high power fields. No significant cytologic atypia or tumour cell necrosis was seen.

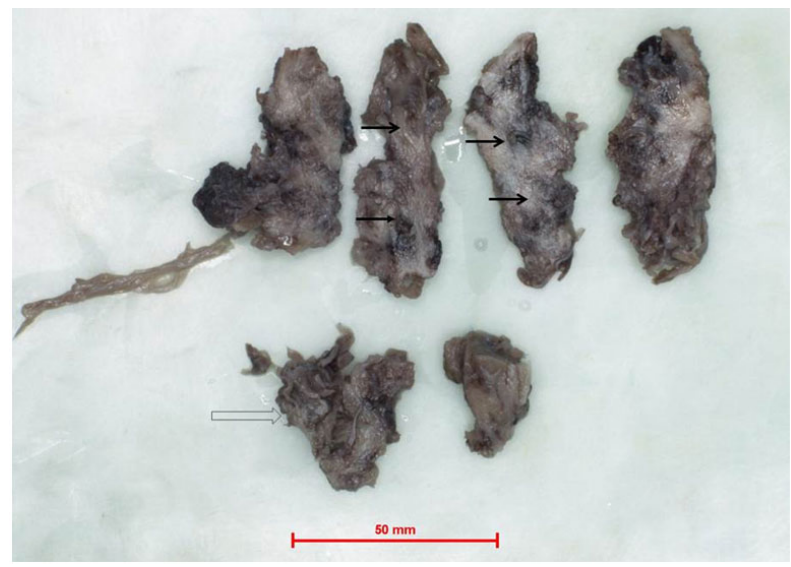

Figure 3. Cut slices of the exophytic component of the tumour. Thin arrows show irregular dissecting borders of the tumour within the myometrial tissue, and open arrows show the placenta-like gross appearance of the tumour.

\section{Discussion}

In the present case, the laboratory was not in possession of a single total hysterectomy specimen. Instead, there were resection specimens of fibroid, uterine wall deposit and myometrium. Despite this, the macroscopic appearance of these specimens, together with the microscopic findings were in good agreement with a cotyledonoid dissecting leiomyoma. Over 30 cases of this tumour have been reported up to this date in the published literature, one of which had described the presence of adenomyosis within the tumour. ${ }^{[3]}$ Uterine cotyledonoid dissecting leiomyomas with intravascular luminal growth, ${ }^{[4]}$ epithelioid variant, ${ }^{[5]}$ hydropic variant ${ }^{[6]}$ and CDL with adipocytic differentiation ${ }^{[7]}$ have also been described. The present case is the second described case in the published literature of a CDL in which non-neoplastic endometrial glands with endometrial stromal cells are present together with an intravascular luminal growth component.

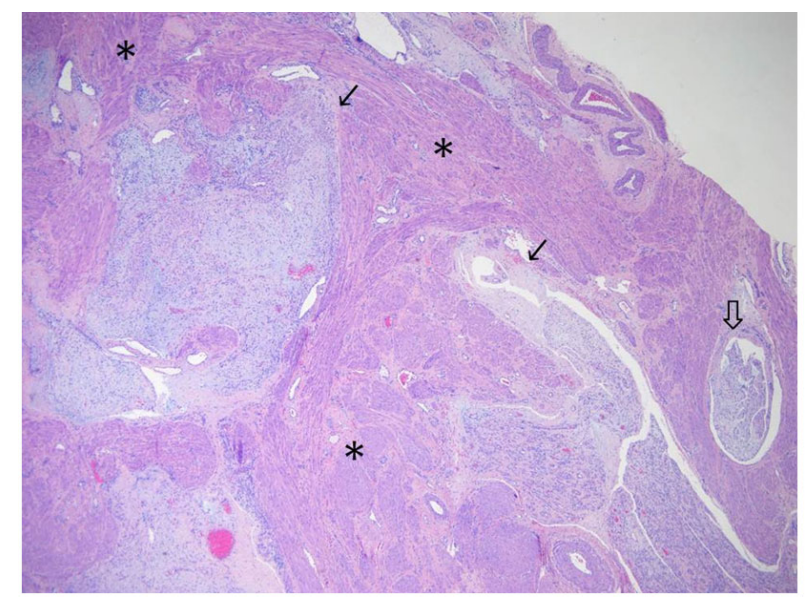

Figure 4. Nodules of tumour (thin arrow) are seen dissecting within the myometrium (asterisks). A focus of intravascular tumour is seen (open arrow). H\&E stain, original magnification $20 \times$.

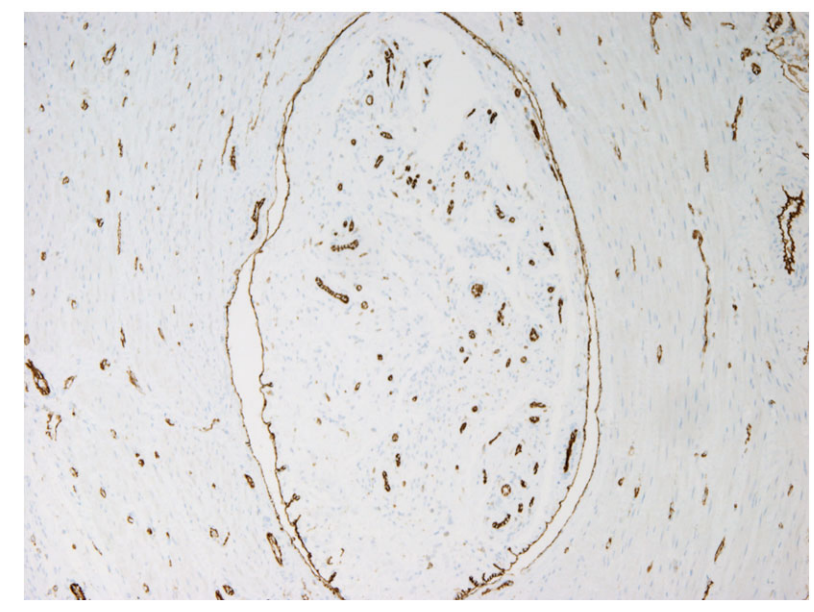

Figure 5. Section of the intravascular leiomyoma within a blood vessel, highlighted by CD31 immunostaining, staining the endothelial cells. Original magnification $40 \times$.

CDLs are seen in women of reproductive or postmenopausal age group ${ }^{[8]}$ and have a wide age range of 23 to 65 years (mean 40.3 years). The majority of patients are admitted to 
hospital with palpable uterine masses and abnormal uterine bleeding. Gynaecological examination reveals a palpable mass in the pelvis that may be detected by ultrasound scan in the uterine corpus or adnexal region. Our patient shows similar clinical features, being 35 years of age, presenting with prolonged and painful menses and showing a mass on ultrasound.

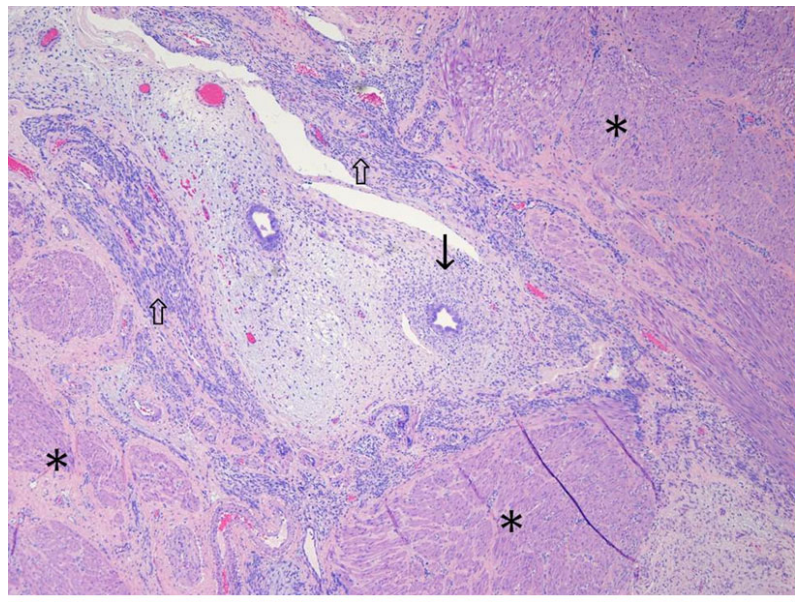

Figure 6. Smooth muscle tumour (open arrow) shows presence of intra-tumoural endometrial glands and stroma (thin arrow). Myometrium is indicated by asterisks. H\&E stain, original magnification $40 \times$.

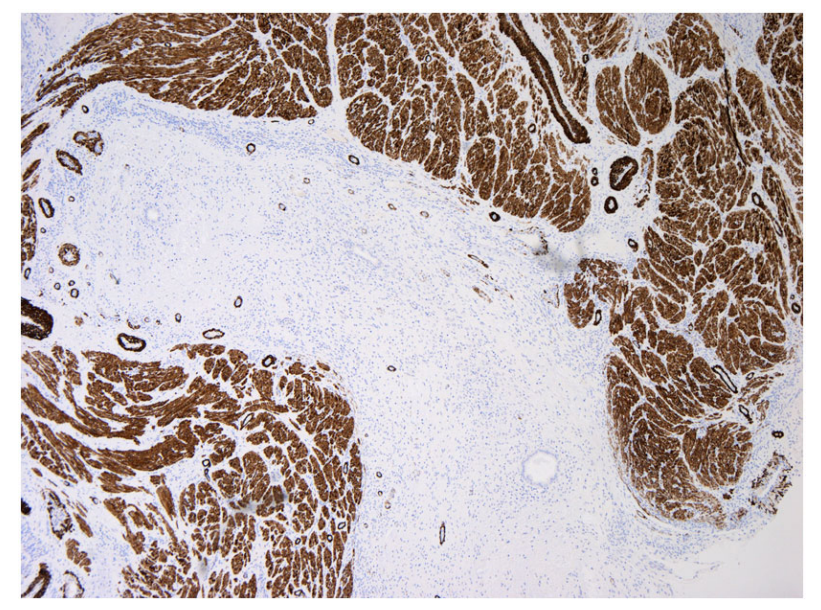

Figure 7. Section of the smooth muscle tumour, showing positive cytoplasmic staining of the neoplastic cells by caldesmon immunostain, original magnification $40 \times$

$\mathrm{CDL}$ is a rare variant of uterine leiomyoma with an unusual macroscopic appearance which resembles a placenta, an unusual growth pattern which may include extra-uterine growth, and which histologically demonstrates an intramural dissecting pattern. Our case showed similar findings, with a soft, cotyledonoid appearance on gross examination, an intraoperative finding of tumour growth onto the uterine serosal surface, and gross and histological demonstration of dissecting masses of neoplastic smooth muscle tissue within the surrounding myometrium. Additional findings within the tumour were areas of oedema and myxoid change. Unlike leiomyosarcoma, the tumour cells show bland cytological appearance, uniform normochromatic nuclei, and minimal mitotic activity. There was no tumour cell necrosis.

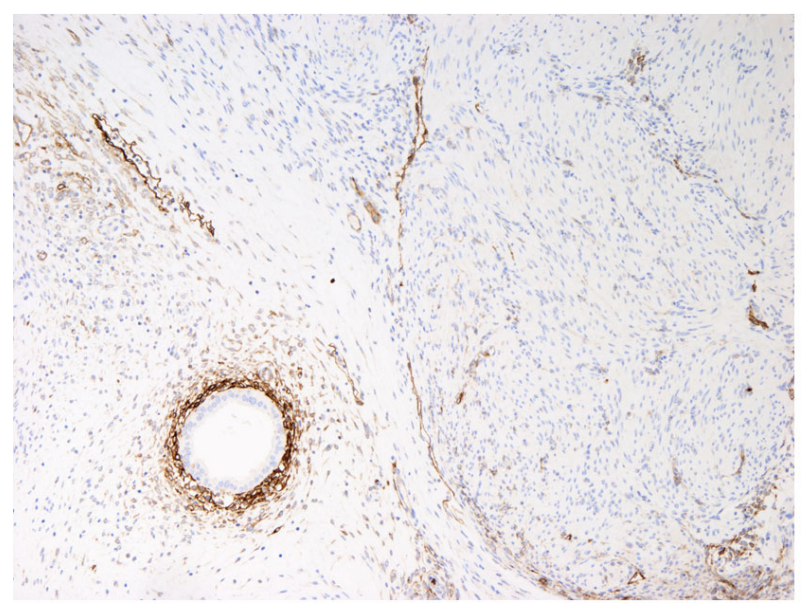

Figure 8. Section of the smooth muscle tumour, showing negative staining of the neoplastic cells for CD10 while the endometrial stromal cells are positive, original magnification $40 \times$

Focally intravascular leiomyomatosis (IVL) was seen, which is uncommon but not unusual in cases of CDL. An intravascular growth pattern has been reported in only one study (in $21.4 \%$ of cases) of CDL. ${ }^{[4]}$ Intravascular leiomyomatosis has a benign outcome, although it can extend into the extra-uterine pelvic veins, inferior vena cava and even up to the right side of the heart. ${ }^{[4]}$ The presence of intravascular growth could be misdiagnosed as a malignancy, such as leiomyosarcoma or endometrioid stromal sarcoma. Unlike leiomyosarcomas, the smooth muscle cells in our case showed no cytological atypia, tumour cell necrosis nor increased mitotic activity. Endometrioid stromal sarcomas (ESS) comprise cells resembling the stromal cells of proliferative endometrium, with minimal ill-defined cytoplasm and oval to fusiform rather than spindle-shaped nuclei. Smooth muscle differentiation may be seen in ESS, but tend to be more focal and patchy. In our case, the tumour cells uniformly showed the appearance of a smooth muscle neoplasm.

What was unusual in our case were the foci of endometrial glands with surrounding stroma within the neoplastic tissue. The localization of the endometrial component was not superficial. Although the possibility of entrapped endometrial tissue was considered, close examination showed that the endometrial glands and stroma in most areas were located 
within the tumour itself. Some foci were present within the tumour-free myometrium, consistent with adenomyosis. Benign uterine adenomyomas are recognized. These are well-circumscribed mixed epithelial and mesenchymal tumours of the uterine corpus, which may show one of two patterns. In one, grossly circumscribed myometrial tumours show endometrial glands and stroma surrounded by hypertrophic smooth muscle fibres in a pattern similar to adenomyosis. In the other, smooth muscle tumours otherwise resembling leiomyomas contain scattered adenomyosis-like foci of glands and stroma within them. Although the tumour in our case showed the latter pattern, it was not well circumscribed, but instead showed a dissecting pattern into surrounding myometrium as well as intravascular growth, resembling that of CDL. During tumour growth from the myometrium, endometrial glands and surrounding stromal cells might have been captured by the intramural component of this tumour during its dissection through surrounding adenomyosis. The presence of endometrial glands in the tumour as well as in the tumour-free myometrium supports this possibility.

The patient was followed up for 6 months since the operation, and was clinically reviewed twice. She remained asymptomatic, with regular menses and no abnormal uterine bleeding. Ultrasound of the uterus was performed twice and showed no recurrence of the tumour.

\section{Conclusions}

Cotyledonoid dissecting leiomyoma is a rare variant of leiomyoma. This case is unique in that it shows the pres- ence of endometrial glands and stroma within the tumour, which may be interpreted as dissecting adenomyoma. The gross and radiological appearance can raise concern for malignancy; however, it is a benign smooth muscle tumour without potential for metastasis or malignant behaviour. Only one case of recurrent CDL following myomectomy has been described. ${ }^{[1]}$ Gynaecologists, radiologists and pathologists should be aware of CDL and its different variants to avoid misdiagnosis and overtreatment, even at the time of intraoperative frozen section evaluation. This unique case with entrapped endometrial glands, stroma, and intravascular invasion reflects the wide spectrum of differentiation within smooth muscle tumours. Although both CDL and IVL are benign, IVL has the potential to spread more extensively, even up to the right side of the heart, whereas CDL spreads more locally and does not usually recur. Hence it is important to distinguish between these two entities.

\section{ACKNOWLEDGEMENTS}

We wish to thank Dr. Esther Oliva, Department of Pathology, Massachusetts General Hospital who has seen this case in consultation. We would also like to thank Dr. Matthias Maiwald, Department of Pathology and Laboratory Medicine, KK Women's and Children's Hospital, Singapore, for helpful suggestions on the manuscript. We also wish to thank the patient for granting us permission to submit this manuscript for publication.

\section{CONFLicts OF INTEREST Disclosure}

The authors declare no conflicts of interest.

\section{REFERENCES}

[1] Roth LM, Reed RJ, Sternberg WH. Cotyledonoid dissecting leiomyoma of the uterus. The Sternberg tumor. Am J Surg Pathol. 1996; 20: 1455-61. PMid: 8944038. https ://doi .org/10.1097/0000 0478-199612000-00004

[2] Smith CC, Gold MA, Wile G, et al. Cotyledonoid dissecting leiomyoma of the uterus: A review of clinical, pathological and radiological features. Int J Surg Pathol. 2012; 20: 330-41. PMid: 22710314. https://doi.org/10.1177/1066896912450315

[3] Shimizu A, Tanaka H, Iwasaki S, et al. An unusual case of uterine cotyledonoid dissecting leiomyoma with adenomyosis. Diagn Pathol. 2016; 11: 69. PMid: 27491369. https://doi.org/10.1186/s1 3000-016-0523-1

[4] Kim NR, Park CY, Cho HY. Cotyledonoid dissecting leiomyoma of the uterus with intravascular luminal growth: a case study. Korean J
Pathol. 2013; 47: 477-80. PMid: 24255637. https ://doi.org/10 .4132/KoreanJPathol.2013.47.5.477

[5] Chawla I, Bhardwaj M, Sareen N, et al. Epithelioid cotyledonoid leiomyoma of uterus. BMJ Case Rep. 2014; pii: bcr2013202434. https://doi .org/10.1136/bcr-2013-202434

[6] Jordan LB, Al-Nafussi A, Beattie G. Cotyledonoid hydropic intravenous leiomyomatosis: a new variant leiomyoma. Histopathology. 2002; 40. https://doi .org/10.1046/j.1365-2559.2002 $.01359 . \mathrm{x}$

[7] Blake EA, Cheng G, Post MD, et al. Cotyledonoid dissecting leiomyoma with adipocytic differentiation: A case report. Gynecol Oncol Rep. 2014; 11: 7-9. https://doi.org/10.1016/j.gore. 2014 . 10.004

[8] Ersöz S, Turgutalp H, Mungan S, et al. Cotyledonoid leiomyoma of uterus: A case report: Turk Patoloji Derg. 2011; 27: 257-60. PMid: 21935878. https://doi.org/10.5146/tjpath. 2011.01085 